\title{
Investigation on the Development Status of Diamond Cutting Tools
}

\author{
G.R. Xie ${ }^{1, a}$, Y.Y. Bao ${ }^{2, b, *}$ \\ ${ }^{1}$ School of Mechanical Engineering, Nantong University, Nantong, Jiangsu, 226019, China \\ ${ }^{2}$ Nantong Li Gang Mechanical Ltd. Nantong, Jiangsu, 226019, China \\ axie.gr@ntu.edu.cn, b442351754@qq.com
}

Keywords: diamond, cutting tool, superhard materials

\begin{abstract}
Precision machining technology, a key technology in modern manufacturing, is widely used in the defense industry, high-tech industry, aerospace and other fields. The advanced cutting tool is an indispensable factor in achieving precision machining technology. And the study on superhard cutting tool with wear-resistant and stable characteristics becomes a hot topic in the universities and scientific research institutes. A lot of studies focus on the diamond tool which is an excellent cutting material with properties of high hardness, low friction coefficient, high thermal conductivity and low thermal expansion coefficient.
\end{abstract}

\section{Introduction}

With the rapid development of the automotive, aviation and aerospace technology, performance and technical requirements for processing of materials are increased. New materials such as carbon fiber reinforced plastics, particle reinforced metal matrix composite and ceramic materials are widely used. These materials have the characteristics of high strength, good abrasion resistance, low thermal expansion coefficient, which determines the life of the cutter in machining time is very short. Development of new wear-resistant and stable superhard cutting tools is the issue that a lot of universities and institutes are focus on.

Diamond tool is the ideal machining tool for high hardness of the material, but also for high-speed precision and automated processing. Now it is widely used in auto parts which require high precision and large quantities. While such tools are expensive than hard alloy, but as long as the processing of objects and conditions are chosen appropriately that the tooling cost will be lower than carbide tools.

CVD diamond films contain no metal or non-metallic additive and its polycrystalline structure in all directions has almost the same high hargydness and has no cleavage plane. Therefore, the overall performance of its physics and machinery combines the advantages of single crystal diamond and polycrystalline diamond. It also overcomes the deficiencies and it is expected to be widely used in precision machining fields.

\section{Characteristics of diamond tool material}

Diamond tools are divided into natural diamond and artificial diamond tools. Natural diamond has the highest hardness and thermal conductivity in natural substances. But it is expensive and difficult to machine, except for a few special purposes such as watches precision parts, optical accessories and jewelry carving processing. It is rarely used as a cutting tool in industry. With the development of high technologies and precision machining, such as nuclear reactors, the navigation gyro of mirrors, computer hard chips, accelerator electron gun and other precision parts processing, natural diamond single crystal can meet the above requirements. In recent years, with the development of a variety of chemical mechanism of the grinding diamond tools and the technology of protective atmosphere brazing diamond, it makes the manufacturing process of natural diamond tools to become simple. Therefore, in the precision mirror machining application, the natural diamond plays an important role.

The hardness and abrasive resistance of diamond is high, and its micro hardness is up to 
$10000 \mathrm{HV}$. At the same time, the processing surface quality is good because of its low friction coefficient and it has no affinity with the non-ferrous metal. It can process non-ferrous metal materials and non-metallic materials, such as copper, aluminum, ceramics, hard alloy, plastic, rubber, graphite, glass and all kinds of wear-resistant wood (especially solid wood and plywood and other composite materials).

Diamond also has disadvantages of poor toughness and low thermal stability. It is easily carbonized at $700^{\circ} \mathrm{C}$, so it is not suitable for processing iron and steel materials. The iron atoms and carbon atoms react easily at high temperature, and diamond is transformed into graphite structure. In addition, it is also worn quickly for cutting with nickel-based alloy.

\section{PCD diamond tool}

PCD is a polycrystalline material made of diamond powder by metal binder such as cobalt in high temperature and high pressure. Although its hardness is lower than natural single crystal diamond, it is a random orientation of the polymerization of the diamond grains, which is isotropic and when used as a tool, it can be used for cutting edge grinding [1, 2]. It is not the same as ND that we must choose the best cleavage plane as the rake face for it. Its cutting edge is not very sensitive to accidental damage, and its wear resistance is also very strong. At the same time, you can maintain a sharp cutting edge for a long time. The service life is generally higher than that of hard alloy (WC base) tool 10 to 500 times, and the source of raw materials of PCD is rich. Meanwhile its price is only one of tens of ND to one of a dozen, and its high performance makes it become the replacement of the traditional WC cutting tool.

The performance of PCD is related to the grain size of diamond and binder. Although the large grain size of PCD has better wear resistance. But it is difficult to make high precision cutting tool because of the poor cutting edge $[3,4]$. On the contrary, the tool made of fine grain PCD has the good quality of edge. In order to improve the toughness and weldability of PCD blade, we usually combine PCD with hard alloy to make composite blade (PCD/CC). The flexural strength of composite blade and carbide are basically the same, and the hardness of the working surface is close to the PCD with good weldability, easy regrinding, low cost. PCD/CC commonly uses the way of brazing or machine clip to make the cutting tool.

It is necessary to point out that the cutting edge and the surface quality of the machined work piece of PCD tool are inferior to that of the ND [5]. And its workability is also very poor and the grinding ratio is small. It is difficult to shape arbitrarily according to the geometry of the tip, and still it can not be easily manufactured indexable PCD inserts with chipbreaker and other geometrically complex products.

\section{CVD diamond tool}

This technology has been applied to the cutting tools since the low pressure chemical vapor deposition (CVD) method develop successfully. Through years of application and research and development, the thick film of CVD diamond welding tool has entered the practical stage. The companies in the United States like the Kennametal, General Motors Corp and Norton have product sales. CVD diamond film coated blade is in the applied research stage, some domestic universities, research institutes and tools manufacturers such as Harbin Institute of Technology, Tsinghua University, Beijing Institute of Technology, Shanghai Jiaotong University and Guangzhou nonferrous Metal Research Institute are stepping up the related products research and development work.

The hot wire method, microwave plasma method and DC plasma jet method are used to made CVD diamond. CVD diamond cutting tools can be roughly divided into two categories: diamond film coated tools and diamond thick film welding tool.

\section{CVD diamond film coated tool}

CVD diamond film cutting tools with the overall coating of diamond surface can be refined into a cutting edge with any shape and geometry [6-8]. Diamond film coated tools are better than that of 
PCD in some aspects. The tool can provide much more complex blade style because all surfaces can be covered with film, and it can lift many limits of the cutting tool depth in the PCD because of the design of broken flute. With the improvement of the coating tool geometry, the tool can still have good performance in high speed machining. However, poor bonding between CVD diamond film and cemented carbide substrate limits the improvement of new technology, and according to the CVD process, the bonding strength between deposition of diamond film coating on the carbide and the matrix is usually lower. There are two reasons: the thermal expansion coefficient is uncoordinated, leading to the interface of diamond and carbide with a larger heat stress; the interaction happens between carbon of the tool surface and the thin layer of cobalt.

In the CVD diamond growth case, cobalt plays a weakening of carbon catalyst formation role, and the substance of this graphite layer is in the role of hindering the bond of diamond to the substrate. The only way to solve the problem is to put a diamond coating in the absence of the adhesive of carbide, which is of no practical value. The wet process is the most successful approach in the practical application, electrochemical or plasma etching technology to get cobalt by corrosion from carbide surface. It is found that cobalt is removed from 5 to $10 \mu \mathrm{m}$ depth range of surface layer without significant decrease of the strength of the matrix, in contrast the strength of the diamond to the surface can be significantly increased. Silicon nitride ceramic is the most suitable matrix material for diamond film coating. Compared with most candidate matrix materials, silicon nitride has very low thermal expansion coefficient, closer to that of diamond, and from the point of view of the chemical properties, silicon nitride ceramic is perfectly suited to the growth of diamond films.

\section{Deposition of thick film diamond tool}

With the rapid development of the technology of diamond deposition, the film is developed into thick film. According to Tokyo Institute of Technology introduces that the deposition rate of diamond is as high as $0.93 \mathrm{~mm} / \mathrm{h}$ [9]. Beijing Oriental Institute of the diamond world was successfully developed a thick film deposition diamond products which deposition speed is much higher than that of current products, and the maximum deposition thickness is reached $2.3 \mathrm{~mm}$. The cost is relatively low, and more importantly, its performance achieved a breakthrough [10,11]. It is isotropic and has no binder, so its hardness is the same as that of natural diamond, much higher than that of PCD and PCBN. From the viewpoint of development, it will be able to replace the PCD, and if its deposition rate and quality are further improved, most of the natural diamond may be substituted.

\section{Conclusions}

Due to its good machining quality and economy, PCD cutting tools shows that other tools can not match advantage in the field of cutting non-metallic materials, non-ferrous metals and alloy material, metal matrix composites. With the theory of PCD cutting tools to further promote the deepening and application of technology, PCD tool position in the field of super-hard tools will become increasingly important, and its scope of application will also be further expanded.

\section{References}

[1] Xue J.X., You S.Y., Yu J.M. (2003) Development and application of polycrystalline diamond (PCD) tool. Tool Technology, 4, 45-47.

[2] Bai Q.S., Yao Y.X. (2002) Development review of polycrystalline diamond (PCD) tool. Tool Technology, 3, 7-10.

[3] Zong W.J., Li D., Wang H.X. (2003) The development of diamond tool technology. China Mechanical Engineering, 13, 1165-1169.

[4] Feng K.M., Wang W., Yin K. (2003) Analysis of development of PCD, PCBN cutting tool. Jewelry Technology, 5, 1-4.

[5] Wu M.J. (2002) Present situation of ultra high speed and ultra high speed cutting machine tool 
technology. New technology and new process, 5, 22-24.

[6] Ye W.C., Cheng W. (2002) New progress of superhard cutting tool materials. WMEM, 1, 6-7.

[7] Zeng Y.H., Liu Z., Luo F.X. (2004) Study on the surface quality of precision cutting of CVD diamond film coated tools. Tool Technology, 7, 9-11.

[8] Zhu J.L., Gou L., Yan S.F. (2004) Research progress of CVD diamond coated carbide tools. Hard alloy, 3, 177-181.

[9] Lv Z.Y., Kang S.J. (2004) Study on manufacturing method of diamond tools. Tool Technology, 2, 17-18.

[10]Yao X.X., Zhang G.X. (2001) Research status and trend of super hard material cutting tools. Hard alloy, 3, 182-186.

[11]Ai X., Liu Z.Q., Zhao J. (2001) Development and future of high speed cutting tool materials. Manufacturing technology and machine tools, 8, 21-25. 